\title{
Tiger snake (Notechis spp) envenoming: Australian Snakebite Project (ASP-13)
}

\section{Geoffrey K Isbister BSC, FACEM, MD, Associate Professor, and Clinical Toxicologist ${ }^{2}$ \\ Margaret A O'Leary \\ Hospital Scientist ${ }^{2}$ \\ Matthew Elliott Medical Student \\ Simon G A Brown MB BS, FACEM, PhD Professor, ${ }^{4}$ and Emergency Physician ${ }^{5}$ \\ 1 Discipline of Clinical Pharmacology, University of Newcastle, \\ Newcastle, NSW. \\ 2 Department of Clinical Toxicology and \\ Pharmacology, Calvary Mater Newcastle Hospital, Newcastle, NSW. \\ 3 School of Medicine University of Melbourne, Melbourne, VIC. \\ 4 Centre for Clinical Research in Emergency Medicine, Western Australian Institute for Medical Research and University of Western Australia Perth, WA \\ 5 Emergency Department, Royal Perth Hospital, Perth, \\ Geoffrey.isbister@ newcastle.edu.au}

MJA 2012; 197: 173-177 doi: 10.5694/mjall.11300

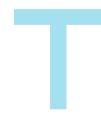

iger snakes are one of the most frequent causes of envenoming in Australia, particularly in Victoria, and are the most important venomous snake in Tasmania. There are two species of tiger snake - Notechis scutatus (mainland tiger snake) and Notechis ater (black tiger snake) — although a recent taxonomic revision suggests the distinction may be somewhat arbitrary. ${ }^{1}$ Mainland tiger snakes average 1.2 metres in length and have significant variability in appearance, from the classical banded dark grey/black and yellow skin to unbanded types with a range of colours from black to light grey. $^{2}$

Most previous reports on tiger snake bites have been case reports of one or a few cases, ${ }^{3-8}$ but there have been two case-series studies, one from South Australia ${ }^{9}$ and another from Western Australia. ${ }^{10}$ Both series were retrospective, with case confirmation based on clinical effects and on a positive result for tiger snake venom using the commercially available snake venom detection kit (sVDK). ${ }^{10}$ The frequency of different envenoming syndromes and the severity of clinical effects in tiger snake envenoming has not been fully characterised.

Tiger snake antivenom (TSAV) was the first snake antivenom to be released by the then Commonwealth Serum Laboratories (which later became CSL Ltd) in 1930. The initial dose was 1 vial. However, over the past few decades, the recommended dose has increased to 4 vials ${ }^{11}$ and, in some cases, many more are given. ${ }^{10,12}$ Recent laboratory and clinical studies

$\begin{array}{ll}\text { Abbreviations } \\ \text { ASP } & \text { Australian Snakebite Project } \\ \text { CK } & \text { Creatine kinase } \\ \text { EIA } & \text { Enzyme immunoassay } \\ \text { INR } & \text { International normalised ratio } \\ \text { SVDK } & \text { Snake venom detection kit } \\ \text { TSAV } & \text { Tiger snake antivenom } \\ \text { VICC } & \begin{array}{l}\text { Venom-induced consumption } \\ \text { coagulopathy }\end{array}\end{array}$

\section{Abstract \\ Objectives: To describe the clinical syndrome associated with definite tiger snake (Notechis spp) envenoming and to examine the ability of tiger snake} antivenom (TSAV) to bind free venom in vivo.

Design, setting and participants: We conducted a prospective cohort study within the Australian Snakebite Project, reviewing all definite tiger snake envenoming cases between October 2004 and June 2011. Definite cases were identified by venom-specific enzyme immunoassay or expert snake identification.

Main outcome measures: Clinical effects of tiger snake envenoming; peak venom concentrations; number of vials of antivenom administered.

Results: Fifty-six definite tiger snake envenomings were identified. Clinical effects included venom-induced consumption coagulopathy (VICC) $(n=53)$, systemic symptoms $(n=45)$, myotoxicity $(n=11)$ and neurotoxicity $(n=17)$. Thrombotic microangiopathy occurred in three patients, all of whom developed acute renal failure. There were no deaths. A bite-site snake venom detection kit test was done in 44 patients, but was positive for tiger snake in only 33 cases. Fifty-three patients received TSAV and eight of these patients had immediate hypersensitivity reactions, severe enough in one case to satisfy diagnostic criteria for severe anaphylaxis. The median peak venom concentration in 50 patients with pretreatment blood samples available was $3.2 \mathrm{ng} / \mathrm{mL}$

(interquartile range [IQR], 1-12 ng/mL; range $0.17-152 \mathrm{ng} / \mathrm{mL}$ ). In 49 patients with post-treatment blood samples available, no venom was detected in serum after the first antivenom dose. Ten patients were given 1 vial of TSAV; the median dose was 2 vials (range, 1-4 vials). Pretreatment serum venom concentrations did not vary significantly between patients given 1 vial of TSAV and those given 2 or more vials.

Conclusion: Tiger snake envenoming causes VICC, systemic symptoms, neurotoxicity and myotoxicity. One vial of TSAV, the dose originally recommended when the antivenom was first made available, appears to be sufficient to bind all circulating venom.

have shown that far less antivenom is required to neutralise the effects of Australian snake venoms ${ }^{13,14}$ and to fully bind venom in vivo. ${ }^{15-17}$

The aims of our study were to report the clinical syndrome that occurs with definite tiger snake envenoming and to examine the ability of TSAV to bind free venom in vivo.

\section{Methods}

We conducted a prospective cohort study of tiger snake envenomings recruited to the Australian Snakebite Project (ASP). The ASP is a prospective multicentre study of suspected snakebites or snake envenomings from over 100 Australian hospitals. The study design, recruitment and data collection have been described previously, ${ }^{15,18}$ and approval has been obtained from 19 human research and ethics committees covering all institutions involved.
Demographic details, clinical information, laboratory results, treatments and reactions are documented on clinical research forms by treating staff. Data are entered into a purposebuilt relational database. Blood samples from each patient are centrifuged and aliquoted, and the serum is stored at $-80^{\circ} \mathrm{C}$ for later analysis of venom concentrations.

\section{Clinical syndromes}

Clinical envenoming syndromes were defined for each patient based on the clinical and laboratory features, using a previously developed classification: venom-induced consumption coagulopathy (VICC) (partial or complete), myotoxicity, neurotoxicity, thrombotic microangiopathy and systemic symptoms. ${ }^{19}$ In complete VICC, there is undetectable fibrinogen and/or a raised D-dimer concentration in citrate plasma (either at least 10 times the assay cut-off point or $>2.5 \mathrm{mg} / \mathrm{L}$ ) and an international normalised ratio 
$(\mathrm{INR})>3.0$. In partial VICC there is a low but detectable fibrinogen level, elevated D-dimer level and a maximum INR <3.0. Myotoxicity is defined as an elevated creatine kinase (CK) level (>1000 U/L) associated with myalgia and/or muscle tenderness.

The presence of any one of these syndromes was considered indicative of systemic envenoming. Clinical features of any hypersensitivity reactions were also documented and were graded according to Brown's scale. ${ }^{20}$

\section{Case identification}

We reviewed all cases from October 2004 to June 2011 and identified possible tiger snake bites or envenoming cases on the basis of expert snake identification, a positive sVDK test for tiger snake venom, or clinical suspicion (in geographical areas where tiger snakes occur). These included cases of VICC, from areas where tiger snakes occur, in which a urine or bitesite sample initially tested positive for brown snake (Pseudonaja spp) venom with sVDK, but was later found to be negative for brown snake venom using serum venom-specific enzyme immunoassay (EIA). Stored samples were analysed for the presence of venoms from snakes known to inhabit the relevant geographical area. Definite tiger snake envenomings were defined by either a serum venom-specific EIA being positive for tiger snake venom and negative for all other relevant venoms or by expert identification of the snake that had bitten a patient who showed features of envenoming.

\section{Antivenom dose}

Antivenom dose was defined as the amount given before the first postantivenom blood collection for the measurement of venom. For patients given antivenom other than tiger snake antivenom, the following conversions were used (based on a previous study that showed that all terrestrial snake antivenoms produced by CSL Ltd are in fact polyvalent ${ }^{21}$ ): 1 vial of brown snake antivenom is equivalent to 0.6 vials of tiger snake antivenom, and 1 vial of polyvalent antivenom is equivalent to 4 vials of tiger snake antivenom. These are the average conversions, and there is variation from batch to batch. $^{21}$

\section{Enzyme immunoassay}

In patients with blood samples available before and/or after antivenom administration, the venom concentration was measured using a venomspecific EIA to detect venoms from Notechis spp (tiger snake), Tropidechis carinatus (rough-scaled snake), Hoplocephalus spp (which includes the broad-headed snake, pale-headed snake and Stephens' banded snake) and Pseudonaja spp (brown snake). Venom-specific antibodies were not available for Austrelaps spp (copperhead snake).

The method uses polyclonal antibodies (IgG) to each venom, raised in rabbits. Detection is by biotinylated antibodies followed by streptavidin horseradish peroxidase, as described elsewhere. ${ }^{17,22}$ The limit of detection for the assay is $0.15 \mathrm{ng} / \mathrm{mL}$ for individual venoms, and the assay is highly specific for each venom, except that Notechis and T. carinatus can only be distinguished from one another at concentrations above $2 \mathrm{ng} / \mathrm{mL}{ }^{16}$ This quantitative and specific EIA differs from the sVDK test, which is a qualitative EIA based on visual inspection of colour changes in wells. The latter is used to rapidly detect venom at the bite site or in urine.

The peak pre-antivenom tiger snake venom concentration was reported for each patient.

\section{Statistical analysis}

For descriptive statistics, median, range and interquartile range (IQR) were calculated for data not normally distributed. Proportions are given with $95 \%$ confidence intervals calculated using the Wilson's procedure with a continuity correction. ${ }^{23}$ Statistical and graphical analyses were done using GraphPad Prism software, version 5.03 for Windows (GraphPad Software Inc, San Diego, Calif, USA).

\section{Results}

From 138 possible tiger snake bites and an additional four cases initially classified as brown snake bites, we identified 56 definite tiger snake envenoming cases and four definite non-envenomed tiger snake bites.
The remaining 78 cases included 39 definite rough-scaled snakebites (based on EIA), 17 probable tiger snake bites (based on geography and sVDK tests without EIA confirmation), 18 cases in which there was either no venom EIA confirmation or the amount of venom detected by EIA was too low to distinguish between tiger snake or rough-scaled snake venom, one definite brown snake bite, and three inconclusive cases in which no venom was detected. Of the 56 definite tiger snake envenomings, the majority occurred in southern Australia and nine involved bites by captive snakes (Box 1).

\section{Clinical effects}

The patient demographic characteristics and clinical effects of the 56 tiger snake envenoming cases are summarised in Box 2 and Box 3. All envenomed patients developed VICC, neurotoxicity or myotoxicity, alone or in combination (Box 4). There was no relationship between the pattern of clinical syndromes and geographical location of the bite. There were no deaths. Five patients had an early collapse, including one associated with a seizure. One of the patients had a cardiac arrest, but also developed features of anaphylaxis before receiving antivenom and had a history of previous snakebites. Systemic symptoms occurred in 45 patients $(80 \%)$, with nausea, vomiting and headache being most common (Box 3).

VICC was the commonest manifestation of envenoming. Thirty-nine patients had complete VICC. In these patients the median time until the INR returned to $<2.0$ was 14.5 hours (IQR, 11.3-18.6 hours). Fourteen patients had partial VICC. Of the remaining three envenomed patients, one presented 8 days after the bite, one had a normal INR and a D-dimer level 10 times the upper limit of normal, and the third had a normal INR but fibrinogen and D-dimer levels were not tested. Bleeding occurred in 17 patients, and two of these had haematemesis without evidence of major gastrointestinal haemorrhage. There were no other major haemorrhages. Thrombotic microangiopathy, which occurred in three patients with VICC, was characterised by acute renal failure, thrombocytopenia and 
1 Distribution of definite tiger snake envenoming cases, including bites by snakes in the wild and captive snakes

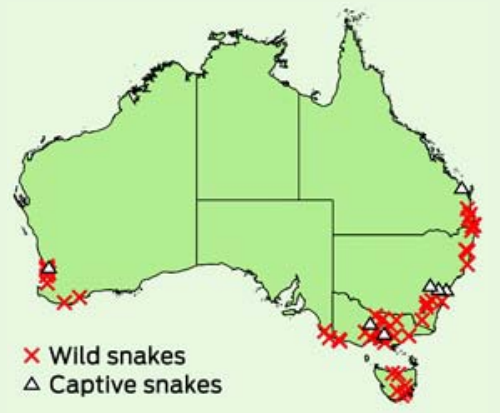

2 Characteristics and demographic features of 56 patients with definite tiger snake envenoming

\begin{tabular}{lc} 
Characteristic/feature & \multicolumn{1}{c}{$\begin{array}{c}\text { Number of } \\
\text { patients* }\end{array}$} \\
\hline $\begin{array}{l}\text { Median age in years } \\
\text { (IQR; range) }\end{array}$ & 42 (29-52; 5-87) \\
$\begin{array}{l}\text { Sex (male) } \\
\text { State or territory } \\
\text { Victoria }\end{array}$ & 41 \\
Western Australia & 18 \\
Queensland & 10 \\
New South Wales & 6 \\
South Australia & 11 \\
Tasmania & 3 \\
Australian Capital & 6 \\
Territory & 2 \\
Bite site & \\
Upper limb & 29 \\
Lower limb & 25 \\
PBI applied & 48 \\
Antivenom & 53 \\
administered & \\
Snake handler & \\
\hline
\end{tabular}

$\mathrm{IQR}=$ interquartile range. $\mathrm{PBI}=$ pressure bandage with immobilisation. *All figures represent number of patients except those relating to median age.

microangiopathic haemolytic anaemia (two of these cases have been reported in detail elsewhere ${ }^{24}$ ).

Neurotoxicity occurred in 17 patients $(30 \%)$ and was severe in one patient, who had bulbar, respiratory and limb weakness requiring intubation and ventilation. The remaining 16 patients had mainly extraocular involvement (Box 3). Four of the 17 patients with neurotoxicity also had myotoxicity. Myotoxicity occurred in 11 patients and there was a raised $\mathrm{CK}$ level (>1000 U/L) in another five. The median peak CK level was $4749 \mathrm{U} / \mathrm{L}$ (range, 1193-203110 U/L). All 11 patients had local muscle pain and tenderness, six developed generalised myalgia or muscle tenderness, and one developed trismus. Acute renal failure did not develop in association with myotoxicity, except in one case where thrombotic microangiopathy was present. The patient with the most severe case of myotoxicity (who also developed severe neurotoxicity) had a peak CK level of $203110 \mathrm{U} / \mathrm{L}$ but did not develop renal failure.

Local pain was reported in 51 patients, with associated bruising in 35 patients and swelling in 33. Of two patients who developed local necrosis, one had an associated secondary infection.

\section{Venom detection kit results}

In 44 of the 56 definite envenoming cases, a bite-site sVDK test was done. Of the 12 patients who did not have a bite-site sVDK test, four had a positive urine sVDK test for tiger snake, four had an expert identification of the snake at the time, and four were in Tasmania, where sVDK tests are not required because tiger snakes and copperhead snakes are the only venomous species present.

The bite-site sVDK test was positive for tiger snake only in 33/44 cases, negative for all species in four cases (subsequent urine sVDK tests were positive for tiger snake in three of these), positive for both brown snake and tiger snake in two cases, and positive for brown snake only in five cases. Of the five patients with a positive sVDK test for brown snake, three received brown snake antivenom, one received 1 vial of brown snake and 2 vials of tiger snake antivenom, and one presented with myotoxicity 8 days after being bitten by a captive snake and did not receive antivenom. In one of the non-envenomed cases involving an identified tiger snake, an sVDK test was positive for brown snake, but a venom-specific EIA on serum was negative for tiger snake venom.

\section{Antivenom treatment}

Of the 56 patients with definite tiger snake envenoming, 53 received antivenom, with a median dose of 2 vials (IQR, $1-2$ vials; range, $1-4$ vials). Eleven of these patients received only 1 vial of TSAV. The remainder received 2 or more vials of TSAV, except for three patients who received brown snake antivenom (2, 2 and 5 vials, respectively). There was no difference in time to receipt of antivenom between patients who developed neurotoxicity and those who did not. Eight patients had systemic hypersensitivity reactions: seven had skin-only reactions (Brown Grade 1) and one had severe anaphylaxis (Brown Grade 3).

Of the three envenomed patients who did not receive antivenom, two had partial VICC alone, and one presented 8 days after being bitten.

\section{Venom assays}

In the 50 patients for whom pretreatment blood samples were available, the median peak venom concentration was $3.2 \mathrm{ng} / \mathrm{mL}$ (IQR, 1-12 ng/ $\mathrm{mL}$; range, $0.17-152 \mathrm{ng} / \mathrm{mL})$. Pretreatment venom concentrations did not differ between patients who subsequently received more than 1 vial of antivenom (median, $3.1 \mathrm{ng} / \mathrm{mL}$; IQR, $0.9-13 \mathrm{ng} / \mathrm{mL}$ ) and patients who received only 1 vial of antivenom (median, $3.7 \mathrm{ng} / \mathrm{mL}$; IQR, 1-8 ng/mL).

In the 49 patients for whom postantivenom blood samples were available — including three given only brown snake antivenom and 10 given only 1 vial of TSAV - the postantivenom blood venom concentration was zero.

\section{Discussion}

Our study showed that tiger snake envenoming is characterised by VICC in almost all cases, neurotoxicity in a third of cases and myotoxicity in a fifth of cases. Major bleeding and thrombotic microangiopathy were uncommon. Bite-site sVDK tests were negative for tiger snake antivenom in a quarter of cases, and the incorrect antivenom was administered in four cases. In the 49 patients for whom post-antivenom blood samples were available, no venom was detected in blood after antivenom administration. This included 10 patients who received only 1 vial of TSAV. Patients given only 1 vial of antivenom had similar pre-treatment serum venom concentrations to patients given more than 1 vial.

The clinical syndrome associated with tiger snake envenoming has 
3 Clinical effects of definite tiger snake envenoming in 56 patients

\begin{tabular}{|c|c|}
\hline Clinical effects & $\begin{array}{c}\text { Number of } \\
\text { patients }\end{array}$ \\
\hline VICC & 53 \\
\hline Complete & 39 \\
\hline Partial & 14 \\
\hline Bleeding & 17 \\
\hline Bite site & 14 \\
\hline Intravenous cannula site & 9 \\
\hline Gums & 2 \\
\hline Haematemesis & 2 \\
\hline Neurotoxicity & 17 \\
\hline Ptosis & 12 \\
\hline Extraocular ophthalmoplegia & 11 \\
\hline Dilated pupils & 5 \\
\hline Myotoxicity & 11 \\
\hline Creatine kinase level >1000 U/L & 16 \\
\hline Systemic symptoms & 45 \\
\hline Nausea & 44 \\
\hline Vomiting & 35 \\
\hline Headache & 34 \\
\hline Abdominal pain & 15 \\
\hline Diaphoresis & 24 \\
\hline Diarrhoea & 7 \\
\hline \multicolumn{2}{|l|}{ Other effects } \\
\hline $\begin{array}{l}\text { Early collapse/ } \\
\text { hypotension }\end{array}$ & 5 \\
\hline Acute renal failure* & 3 \\
\hline Thrombotic microangiopathy & 3 \\
\hline Change in taste & 4 \\
\hline
\end{tabular}

VICC $=$ venom-induced consumption coagulopathy. *All three cases of acute renal failure were in association with thrombotic microangiopathy. been previously based on expert anecdotal reports and case reports. Although our results are generally consistent with current understanding of tiger snake envenoming, they provide an accurate description of tiger snake envenoming in a series of patients treated with early antivenom therapy. Tiger snake and rough-scaled snake envenoming syndromes were previously regarded as similar. Our results show that neurotoxicity is more common with tiger snake envenoming, occurring in almost a third of cases, compared with two out of 24 cases of rough-scaled snake envenoming reported in a previous study. ${ }^{16}$

Scop and colleagues reported on a 16-year retrospective series of 23 tiger snake envenoming cases. ${ }^{10}$ However, their inclusion criteria were based on a positive sVDK test, which we have shown here to be unreliable and thus unsuitable for the purpose of case definition in research studies. In addition, Scop and colleagues included only cases in which fibrinogen was undetectable, which biased the series towards patients with complete VICC. ${ }^{10}$ It is recognised that VICC can occur with only partial coagulation factor consumption, ${ }^{25}$ and in our study almost a third of patients with tiger snake envenoming had only partial VICC. An earlier series reported by White described 10 cases of tiger snake envenoming in which there was a similar range of effects. ${ }^{9}$

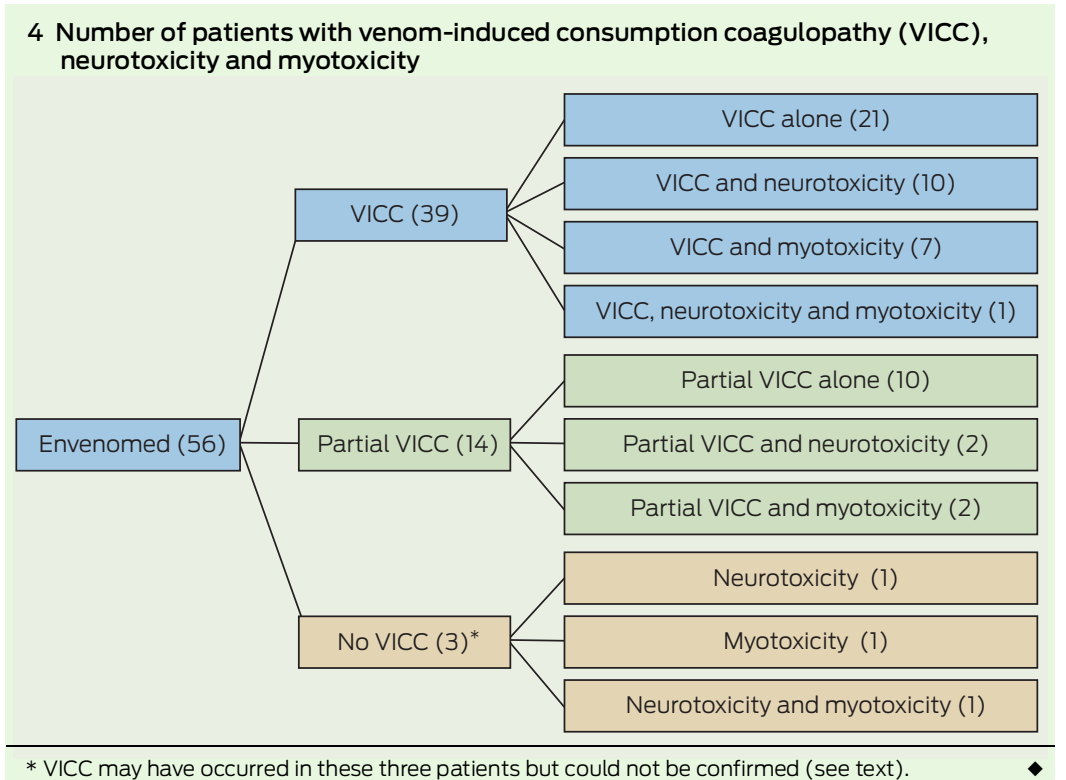
neurotoxicity and myotoxicity

* VICC may have occurred in these three patients but could not be confirmed (see text).
A possible limitation of our study was that serum samples were not tested for all elapid snake venoms, and potentially related snakes such as copperheads (Austrelaps spp) could not be excluded as being responsible for bites where their distribution overlaps that of tiger snakes. However, reports of bites by identified Austrelaps spp are exceedingly rare, and there was no geographical variation in the clinical syndromes reported for tiger snake envenoming that occurred in areas where Austrelaps spp are found compared with other areas.

Our study brings into question the reliance on the sVDK test for determining appropriate antivenom treatment. The sVDK result was incorrect in $5 / 44$ cases, giving a positive result for brown snake venom, which led to the incorrect use of antivenom in four of the five cases. The use of sVDK is thus problematic and may confuse clinical assessment. A "saving grace" in this situation is that, because all commercial antivenoms are polyvalent, ${ }^{21}$ brown snake antivenom contains sufficient TSAV to neutralise the procoagulant activity of tiger snake venom. ${ }^{26}$ However, this principle cannot be extended to the treatment of bites from other snakes that need much higher-volume antivenoms, such as the mulga snake, death adder and taipan. Although all commercial antivenoms are polyvalent, the low-volume monovalent antivenoms (for brown and tiger snake bites) may contain insufficient antivenom to treat bites from snakes that require higher-volume antivenoms. Knowledge about medically important snakes that occur in a region and the clinical syndromes they cause is required for the selection of appropriate antivenom.

The data presented here provide further evidence that 1 vial of antivenom is sufficient to treat envenoming, as initially recommended by CSL Ltd. Larger doses of antivenom have been recommended up until recently, based on in-vitro ${ }^{27}$ and clinical $^{10}$ studies. An in-vitro study used venom concentrations that were 10000 -fold those seen in human snake envenoming. The study by Sprivulis and colleagues ${ }^{27}$ used a serum tiger snake venom concentration of $33 \mu \mathrm{g} / \mathrm{mL}$, compared with the 
median serum concentration of $3.2 \mathrm{ng} / \mathrm{mL}$ found in our study. It is therefore not surprising that this study suggested that larger doses of antivenom were required. Previous clinical studies may have had unrealistic expectations about the time to recovery of clotting function and have reported the amount of antivenom given to patients, rather than determining the amount required to bind all antivenom in vivo, thus overestimating the required dose of antivenom. Our study provides invivo support for the claim that 1 vial of antivenom is an adequate dose. This is consistent with studies on other snakes ${ }^{15-17}$ and with in-vitro studies. ${ }^{14}$

Acknowledgements: We would like to thank the ASP clinical investigators who recruited patients to the study: Yusuf Nagree (Armadale Hospital and Fremantle Hospital), Adam Coulson and Garry Wilkes (Bunbury Hospital), Nick Buckley (The Canberra Hospital), Alan Tankel (Coffs Harbour Hospital), David Spain (Gold Coast Hospital), Andrew Dawson (Royal Prince Alfred Hospital), Colin Page (Princess Alexandra Hospital), Ovidu Pascui (Sir Charles Gairdner Hospital), Naren Gunja (Westmead Hospital), and the ASP laboratory investigators. We also acknowledge the many referrals from poisons information centres and clinical toxicologists and the help of many other nurses, doctors and laboratory staff in recruiting patients and collecting samples.

Competing interests: No relevant disclosures.

\section{Received 8 Oct 2011, accepted 1 Mar 2012}

1 Rawlinson PA. Taxonomy and distribution of the Australian tiger snakes (Notechis) and copperheads (Austrelaps) (Serpentes: Elapidae). Proc R Soc Victoria 1991; 103: 125-135.

2 Cogger HG. Reptiles and amphibians of Australia. Sydney: Reed New Holland, 2000.

3 Tibballs J, Henning RD, Sutherland SK, Kerr AR. Fatal cerebral haemorrhage after tiger snake (Notechis scutatus) envenomation. Med J Aust 1991; 154: 275-276.
4 Sutherland SK, Coulter AR. Three instructive cases of tiger snake (Notechis scutatus) envenomation - and how a radioimmunoassay proved the diagnosis. Med J Aust 1977; 2: 177-180.

5 Penington A, Johnstone B. A case of local tissue necrosis following a bite by the Australian tiger snake Notechis scutatus. Aust NZ J Surg 1997; 67: 385-388.

6 Ferguson LA, Morling A, Moraes C, Baker R. Investigation of coagulopathy in three cases of tiger snake (Notechis ater occidentalis) envenomation. Pathology 2002; 34: 157-161.

7 Hood VL, Johnson JR. Acute renal failure with myoglobinuria after tiger snake bite. Med J Aust 1975; 2: 638-641.

8 Harvey PM, Tabrett DG, Solomons BJ, Thomas MA. Envenomation by a King Island tiger snake (Notechis ater humphreysi). Med J Aust 1982; 2: 192-193.

9 Scop J, Little M, Jelinek GA, Daly FF. Sixteen years of severe tiger snake (Notechis) envenoming in Perth, Western Australia. Anaesth Intensive Care 2009; 37: 613-618.

10 White J. A review of 105 cases of suspected snakebite in South Australia. In: Gopalakrishnakone P, Tan C, editors. Progress in venom and toxin research. Singapore: National University of Singapore, 1987: 15-19.

11 White J.CSL antivenom handbook. Melbourne: CSL, 2001.

12 Parkin JD, Ibrahim K, Dauer RJ, Braitberg G. Prothrombin activation in eastern tiger snake bite. Pathology 2002; 34: 162-166.

13 Isbister GK, Woods D, Alley S, et al. Endogenous thrombin potential as a novel method for the characterization of procoagulant snake venoms and the efficacy of antivenom. Toxicon 2010; 56: 75-85.

14 Isbister GK, O'Leary MA, Schneider JJ, et al. Efficacy of antivenom against the procoagulant effect of Australian brown snake (Pseudonaja sp.) venom: in vivo and in vitro studies. Toxicon 2007; 49: 57-67.

15 Churchman A, O'Leary MA, Buckley NA, et al. Clinical effects of red-bellied black snake (Pseudechis porphyriacus) envenoming and correlation with venom concentrations: Australian Snakebite Project (ASP-11). Med J Aust 2010; 193: 696-700.

16 Gan M, O'Leary MA, Brown SG, et al. Envenoming by the rough-scaled snake (Tropidechis carinatus): a series of confirmed cases. Med J Aust 2009; 191: 183-186.

17 Kulawickrama S, O'Leary MA, Hodgson WC, et al. Development of a sensitive enzyme immunoassay for measuring taipan venom in serum. Toxicon 2010; 55: 1510-1518.

18 Isbister GK, Brown SG, MacDonald E, et al; Australian Snakebite Project Investigators. Current use of Australian snake antivenoms and frequency of immediate-type hypersensitivity reactions and anaphylaxis. Med J Aust 2008; 188 473-476.

19 Isbister GK, White J, Currie BJ, et al; ASP Investigators. Clinical effects and treatment of envenoming by Hoplocephalus spp. snakes in Australia: Australian Snakebite Project (ASP-12). Toxicon 2011; 58: 634-640.

20 Brown SG. Clinical features and severity grading of anaphylaxis. J Allergy Clin Immunol 2004; 114 371-376.

21 O'Leary MA, Isbister GK. Commercial monovalent antivenoms in Australia are polyvalent. Toxicon 2009; 54: 192-195.

22 O'Leary MA, Isbister GK, Schneider JJ, et al. Enzyme immunoassays in brown snake (Pseudonaja spp.) envenoming: detecting venom, antivenom and venom-antivenom complexes. Toxicon 2006; 48: 4-11.

23 Newcombe RG. Two-sided confidence intervals for the single proportion: comparison of seven methods. Stat Med 1998; 17: 857-872.

24 Casamento AJ, Isbister GK. Thrombotic microangiopathy in two tiger snake envenomations. Anaesth Intensive Care 2011; 39: $1124-1127$.

25 Isbister GK, Scorgie FE, O'Leary MA, et al; ASP Investigators. Factor deficiencies in venominduced consumption coagulopathy resulting from Australian elapid envenomation: Australian Snakebite Project (ASP-10). J Thromb Haemost 2010; 8: 2504-2513.

26 O'Leary MA, Schneider J, Krishnan BP, et al. Cross-neutralisation of Australian brown and tiger snake venoms with commercial antivenoms: cross-reactivity or antivenom mixtures? Toxicon 2007; 50: 206-213.

27 Sprivulis P, Jelinek GA, Marshall L. Efficacy and potency of antivenoms in neutralizing the procoagulant effects of Australian snake venoms in dog and human plasma. Anaesth Intensive Care 1996; 24: 379-381.

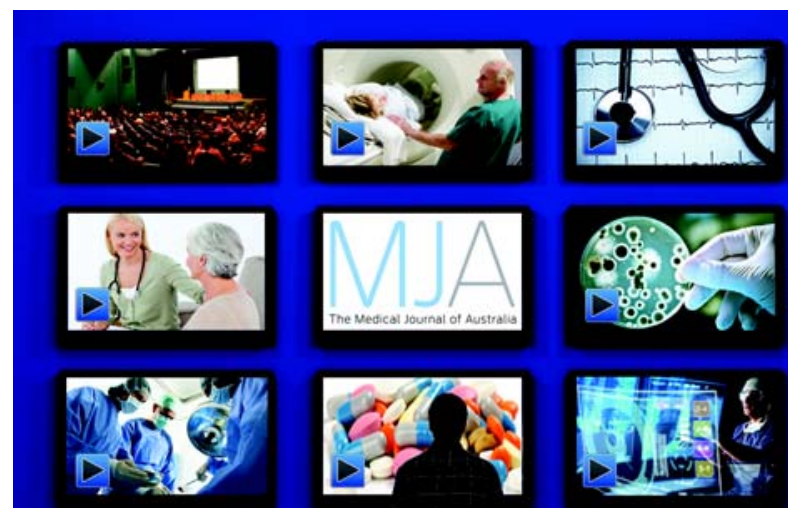

\section{MJA multimedia presentations}

The MJA invites authors to submit video and audio presentations relevant to the practice of clinical medicine in Australia for consideration for publication on the MJA website.

Multimedia presentations will be peer reviewed. Those considered to be of sufficient quality, academic rigour and relevance will be posted on the MJA website, with a brief summary being published in the MJA (citable in indexing services such as PubMed) linking to the presentation.

Video or audio presentations can be made specifically for the MJA or can be lectures, seminars or conference presentations (eg, clinical updates, demonstrations of clinical signs, or techniques) 